\title{
REPETITIVE OVARIAN TORSION AS WARNING SIGN OF SEROUS AND MUCINOUS CYSTADENOMA IN CHILDREN - REPORT OF TWO CASES
}

\author{
Usiwoma Okeroghene Ataikiru ${ }^{1,2}$, Emil Radu Iacob ${ }^{1,2}$, Călin Marius Popoiu ${ }^{1,2}$, Rodica Heredea ${ }^{3}$, \\ Corina Stănciulescu ${ }^{1,2}$, Eugen Sorin Boia ${ }^{1,2}$
}

Abstract

Cystadenomas are surface epithelial-stromal tumors. Although they are the most common type of ovarian tumor, seen mostly from late adolescent age, yet, they remain difficult to accurately diagnose clinically due to their diverse presentations similar to other diseases like functional cyst and appendicitis. We report the cases of two pediatric patients. The first one aged seventeen years and six months old with serous cystadenoma and the second patient aged seventeen years and three months old with mucinous cystadenoma, presenting with abdominal pain in the level of the right iliac fossa and abdominal pain in the inferior part of the abdomen respectively aiming to reveal some warning signs and particularities in diagnosis and management of female pediatric patients with ovarian cystadenomas. Conclusion: Recurrent ovarian torsion or recurrent cyst formation is a warning sign of mucinous cystadenoma and serous cystadenoma tumor requiring further investigations and treatment.

Keywords: serous cystadenoma, mucinous cystadenoma, ovarian torsion, recurrent cyst, pediatric patients

\section{Introduction}

Ovarian tumors are neoplasm found in the ovary and rarely seen in children, with 2.6 per 100,000 children yearly [1]. Cystadenoma ovarian tumors are usually benign in children but malignant in $10-20 \%$ of pediatric patients $[2,3]$. These patients usually present with pelvic and abdominal pain in diverse forms, vomiting, bloating, loss of appetite, vaginal bleeding, increased abdominal volume, constipation, or sometimes fever [4], which are similar to patients with appendicitis or a functional cyst; hence it can be quite challenging to diagnose accurately, especially if not large in size or a benign tumor but with radiological, laparoscopic and histopathology investigations, a specific diagnosis can be made to a large extent. We present the cases of two girls with mucinous cystadenoma and serous cystadenoma treated by surgical tumoral excision.

Case 1

A 17 years and six months old girl presented at our hospital with complaints of abdominal pain in the right iliac fossa and nausea. She had no previous significant medical history and she had a regular menstrual cycle. On physical examination, she weighed $52 \mathrm{~kg}$ and had a height of $160 \mathrm{~cm}$, afebrile, abdominal pain in the right iliac fossa on superficial and deep palpation, without any palpable mass. The number of leukocytes was $8,81 \times 10^{\wedge} 3 / \mathrm{ul}$ and C-reactive protein $2.71 \mathrm{mg} / \mathrm{l}$, urinalysis, and tumor markers were normal. Abdominal ultrasound showed a cyst of $5.27 / 5.1 \mathrm{~cm}$ on the right ovary, but after admission, the pain disappeared, and we placed the patient on paracetamol. Three days later, she was discharged with the diagnosis of a right ovarian cyst. The patient returned after five months complaining of pain at the inferior abdominal level, nonresponsive to antalgic medication, number of leukocytes was $6,37 \times 10^{\wedge} 3 / \mathrm{ul}$, C-reactive protein was $0,15 \mathrm{mg} / 1$, and other laboratory results were normal. Abdominal ultrasound showed the presence of a gigantic abdominal pelvic tumor with dimensions of $15.73 / 8.04 \mathrm{~cm}$, uniloculated, originating from the left ovary (Figure 1). The thoracic $\mathrm{x}$-ray was normal. We performed exploratory laparoscopy and cystectomy and placed the patient on antalgic and antiinflammatory drugs. The histopathological result from the biopsy showed benign serous cystadenoma, irregular cystic fragment, containing clear liquid with whitish - caffein color and elastic consistency, unilocular. The cystic wall lined with simple cylindric ciliated epithelium similar to the fallopian tube epithelium (Figure 2). With a favourable evolution, the patient was discharged after three days.

${ }^{1}$ Department of Pediatric Surgery and Orthopedics "Victor Babes" University of Medicine and Pharmacy Timisoara, Romania.

${ }^{2}$ Department of Pediatric Surgery and Orthopedics "Louis Turcanu" Emergency Children's Hospital Timisoara, Romania.

${ }^{3}$ Department of Pathological Anatomy "Louis Turcanu" Emergency Children's Hospital Timisoara, Romania

E-mail: usiwomaataikiru@yahoo.com, radueiacob@umft.ro, mcpopoiu@yahoo.com,

heredea.rodica@yahoo.com, stanciulescucorina@yahoo.com, boiaeugen@yahoo.com 


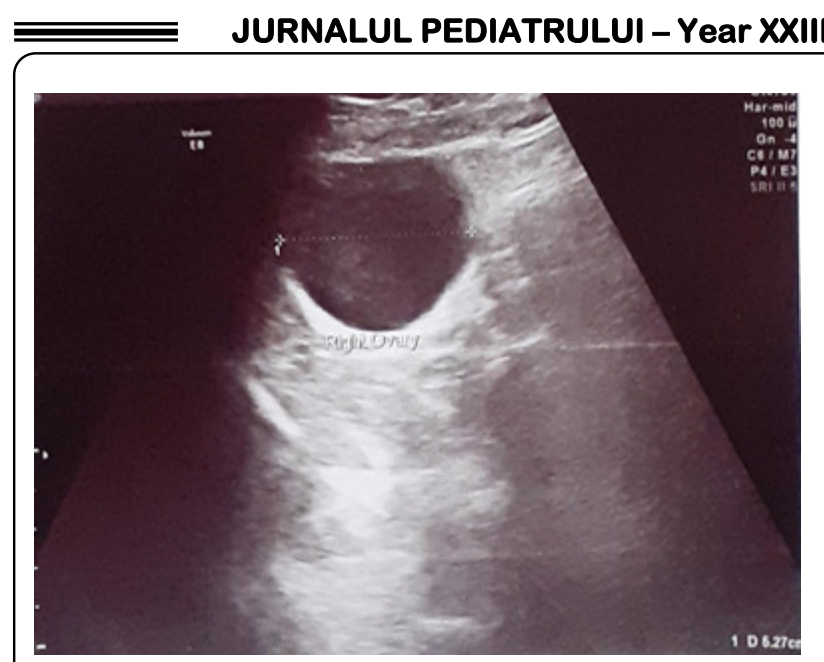

Figure 1. Ultrasound of the right ovary.

\section{Case 2}

A 16 year-old girl presents with colic abdominal pain at the inferior abdominal level that debuted four days ago. Se had loss of appetite and recurrent vomiting episodes. She was unresponsive to antalgic and antiemetic treatment. The patient had a medical history of repetitive biliary colic and irregular menstruation. On physical examination, she weighs $72 \mathrm{~kg}$ and has height of $165 \mathrm{~cm}$ with a BMI of 26,47 ; obese and with dental caries. The number of leucocytes was $8,0 \times 10^{\wedge} 3 / \mathrm{ul}$ and C-reactive protein was $0,45 \mathrm{mg} / 1$, with normal urinalysis. At superficial and deep palpation the patient accused abdominal pain in the left flank and at the peri-umbilical level. Abdominal ultrasound showed transonic ovary of $5 / 3.5 \mathrm{~cm}$, no vascularization present, hence we diagnose the patient of torsion of the left ovary in remission. After two days of treatment, with a favourable condition, she was discharged. Seven months later the patient returned to our hospital, weighing $68 \mathrm{~kg}$,

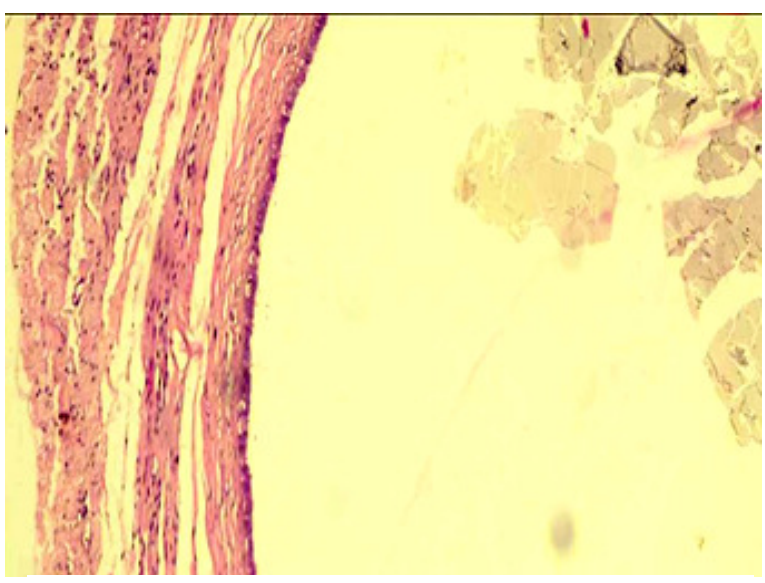

Fig 2. Histopatological aspect of the cystadenoma. Hematoxylin-Eosin stains 20X.

complaining of abdominal pain in the right iliac fossa. Laboratory analysis showed that the number of leucocytes was $6.67 \times 10^{\wedge} 3 / \mathrm{ul}$ and C-reactive protein $0,19 \mathrm{mg} / \mathrm{l}$.

Urinalysis indicated urinary tract infection. The abdominal ultrasound revealed a second torsion of the left ovary, $14 / 12,5 \mathrm{~cm}$, and cyst of the fallopian tube $9 / 7,5 \mathrm{~cm}$. Exploratory laparoscopy showed $360^{\circ} \mathrm{C}$ torsions of left ovary (Figure 3). Detorsion of the ovary and excision of the ovarian cyst was performed and the resected mass was sent for histopathological examination. The result revealed mucinous cystadenoma of the ovary. The cystic wall is lined by an epithelium mucinous columnar, unilocular nuclei at the basal pole, fibrous stroma, ovarian parenchyma attached to a primordial, cystic follicle, and papillary projections (Figure 4). The patient was placed on antalgic and anti-inflammatory treatment. She spent a day in the intensive care unit. Two days later, the patient had a favourable evolution and was discharged.

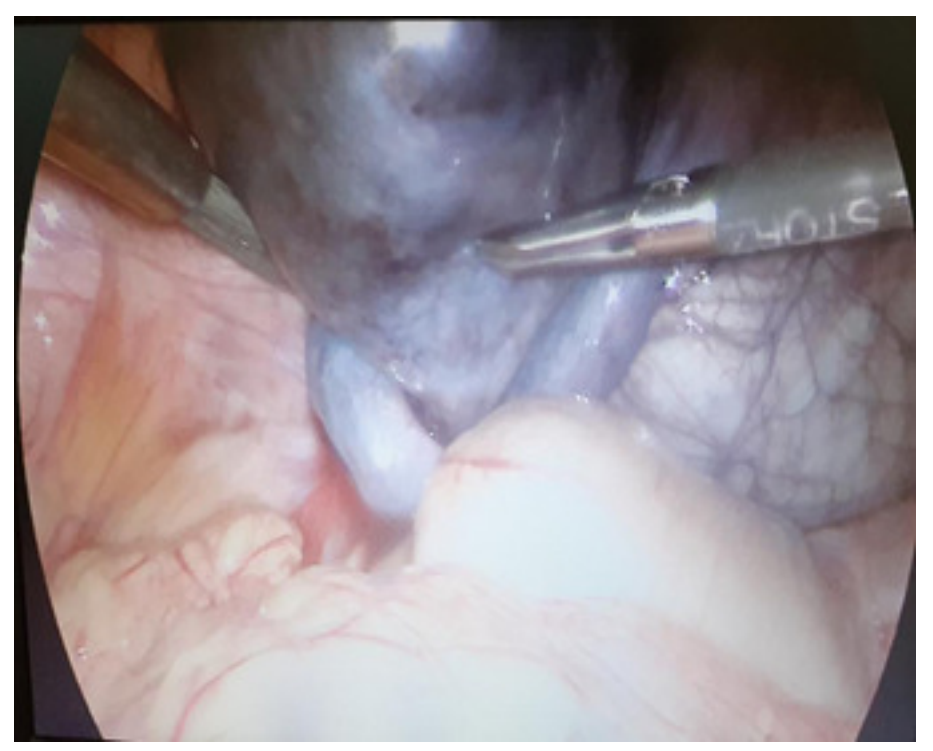

Figure 3. Intra operative image of the torsioned ovary. 


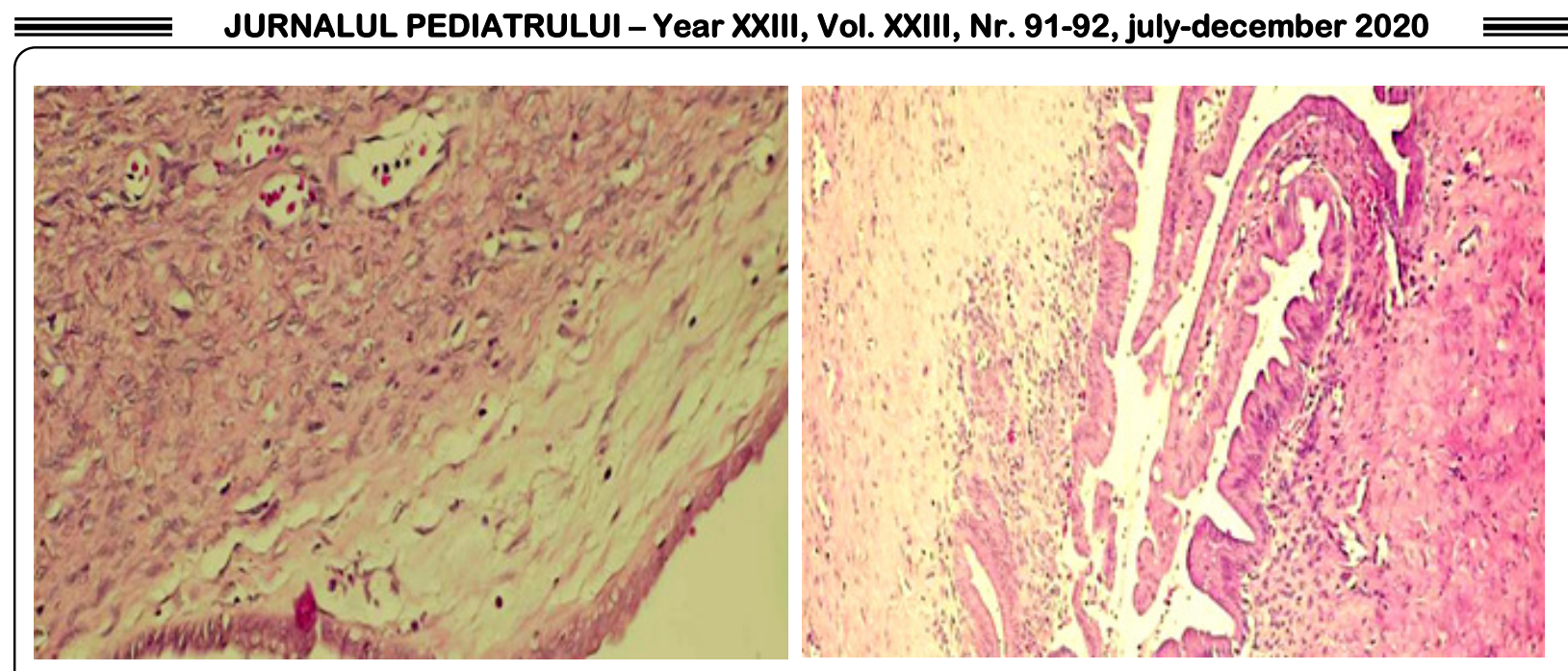

Figure 4. Mucinous cystadenoma of the ovary, endocervical subtype. Hematoxylin-Eosin stain 20X.

\section{Discussion}

Cystadenomas are epithelial tumors representing about $60 \%$ of the ovarian tumors. They can be serous, mucinous, clear cell or endometrioid. Also, they can be benign, borderline, or malignant tumors and can also be cystic, solid, or mixed form [2]. Both patients had abdominal pain in the right iliac fossa, left flank and at peri-umbilical level, loss of appetite, and vomiting (which can be a sign of appendicitis), but neither of them had fever nor an increase in number of leukocytes [4]. Unlike functional cyst, which is unharmful and can self-resolve, cystadenoma has the potential to transform into malignancy as reported in the literature, especially if it is borderline [5-7]. Hence differentiating them is crucial and a method to achieve this accurately and easily is needed. Ovarian torsion is usually found in females of reproductive age, especially in the presence of a mass $>5 \mathrm{~cm}$, which can be a cyst or benign tumor and in rare cases a malignant tumor [8]. The incidence rate of ovarian torsion is estimated to be 4.9: 100000 persons and requires immediate surgical intervention to detorsion the ovary. It rarely re-occurs as a tumor diagnosis, and one of such few cases reported was in a ten years old premenarchal girl [9]. Cyst re-occurrence has been attributed to incomplete excision or infection [10-11], but we see that it can also suggest a tumor's presence. Overweight was another observation noticed in both patients, which is recognized as a contributing risk factor of tumors. Histopathological examination remains the efficient diagnostic method used. Surgical treatment is the standard management for mucinous a serous cystadenoma tumor ranging from cystectomy to gonadectomy. Depending on if it is a malignant cystadenoma, or large tumor, or if the ovary is invaded, then it is difficult to preserve the gonads, but ovary-sparing surgery is the choicest option when possible.

\section{Conclusion}

Irregular menstruation, loss of appetite, a normal laboratory result in an afebrile patient can be used to rule out appendicitis. However, a patient presenting with recurrent ovarian torsion or recurrent cyst formation is a warning sign of mucinous and serous cystadenoma requiring further investigations and the appropriate treatment.

\section{Conflicts of interest}

All authors have no conflicts of interest to declare.

\section{References}

1. Péroux E, Franchi-Abella S, Sainte-Croix D, et al. Ovarian tumors in children and adolescents: a series of 41 cases. Diagnostic and Interventional Imaging. 2015 Mar 1;96(3):273-82.

2. Limaiem F, Mlika M. Treasure Island, FL: StatPearls Publishing; 2020. Ovarian Cystadenoma

3. Elizabeth Kehr, Thing RindaSoong, Michelle S. Hirsch. Chapter 28 - Metastatic Tumors Involving the Ovary. In 2018 Christopher P. Crum, Marisa R. Nucci, Brooke E. Howitt, Scott R. Granter, Mana M. Parast, Theonia K. Boyd (Eds). Third Edition. Diagnostic Gynecologic and Obstetric Pathology. Elsevier, 2018, Pages 1050-1069. ISBN 9780323447324.
4. Bankhead CR, Kehoe ST, Austoker J. Symptoms associated with diagnosis of ovarian cancer: a systematic review. BJOG.2005;112:857-865.

5. Zheng J, Benedict WF, Xu HJ, et al. Genetic disparity between morphologically benign cysts contiguous to ovarian carcinomas and solitary cystadenomas. J Natl Cancer Inst. 1995;87:1146-1153.

6. Fischerova D, Zikan M, Dundr P, Cibula D. Diagnosis, treatment, and follow-up of borderline ovarian tumors. The oncologist. 2012 Dec;17(12):1515.

7. Puls LE, Powell DE, DePriest PD, Gallion HH, Hunter JE, Kryscio RJ, van Nagell Jr JR. Transition from benign to malignant epithelium in mucinous and serous 


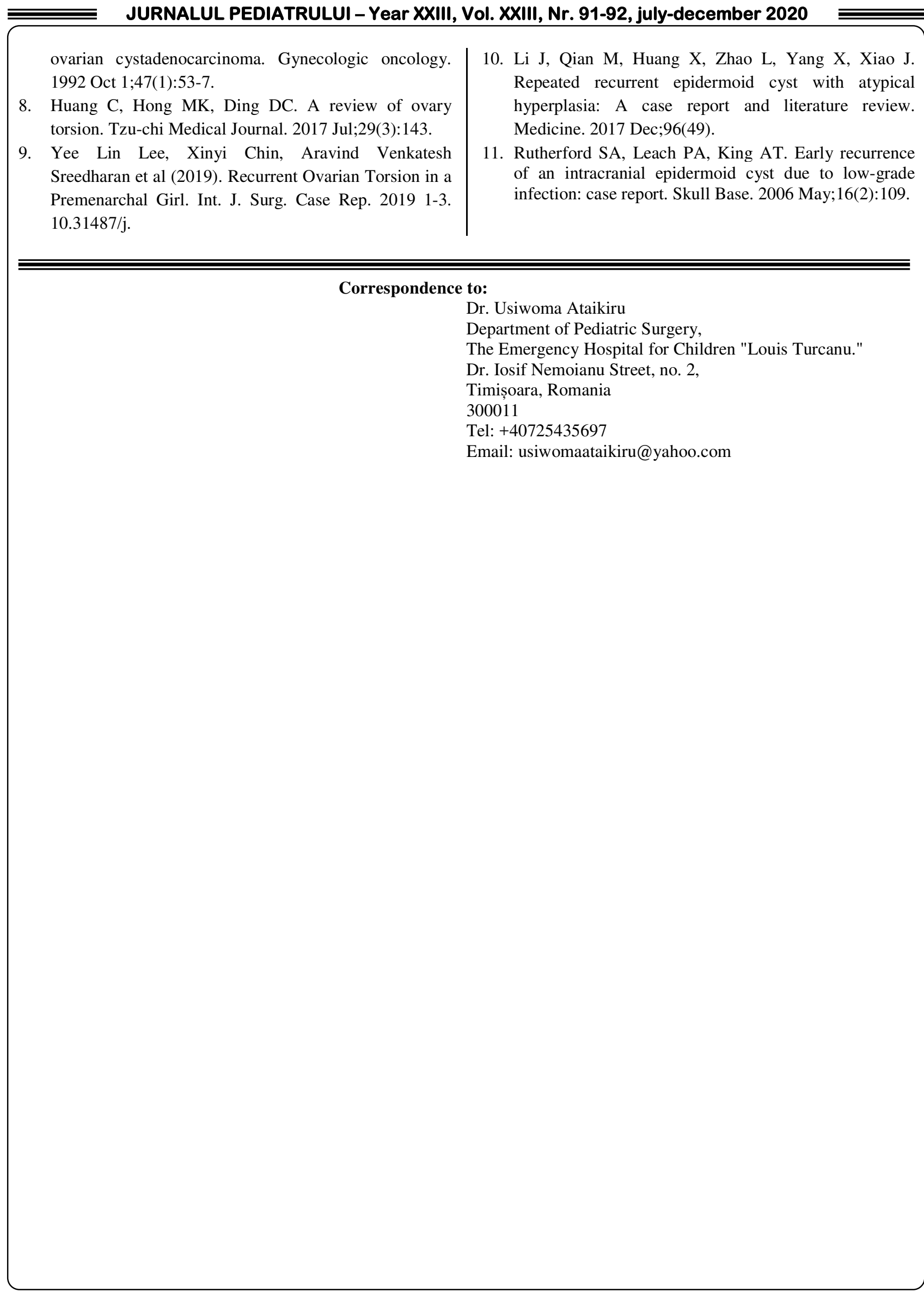

